\title{
MicroRNA-148b promotes proliferation of hair follicle cells by targeting NFAT5
}

\author{
Wanbao YANG, Qinqun LI, Bo SU, Mei YU (凶) \\ Key Lab of Agricultural Animal Genetics, Breeding and Reproduction of Ministry of Education, \\ College of Animal Science and Technology, Huazhong Agricultural University, Wuhan 430070, China
}

\begin{abstract}
MicroRNAs (miRNAs), small non-coding RNAs, are involved in many aspects of biological processes. Previous studies have indicated that miRNAs are important for hair follicle development and growth. In our study, we found by qRT-PCR that miR-148b was significantly upregulated in sheep wool follicle bulbs in anagen phase compared with the telogen phase of the hair follicle cycle. Overexpression of miR-148b promoted proliferation of both HHDPC and HHGMC. By using the TOPFlash system we demonstrated that miR-148b could activate $\mathrm{Wnt} / \beta$-catenin pathway and $\beta$-catenin, $c y c D, c$-jun and PPARD were consistently upregulated accordingly. Furthermore, transcript factor nuclear factor of activated $T$ cells type 5 (NFAT5) and $W n t 10 b$ were predicted to be the target of miR-148b and this was substantiated using a Dual-Luciferase reporter system. Subsequently NFAT5 was further identified as the target of miR-148b using western blotting. These results were considered to indicate that miR- $148 \mathrm{~b}$ could activate the $\mathrm{Wnt} / \beta$-catenin signal pathway by targeting NFAT5 to promote the proliferation of human hair follicle cells.
\end{abstract}

Keywords miR-148b, hair follicle, proliferation, NFAT5, Wnt $10 b$

\section{Introduction}

Hairs, unique to mammals, are organs found in the skin and have various functions ${ }^{[1,2]}$. Unlike other organs during the postnatal life, hair follicles undergo a cyclic period including growth (anagen), regression (catagen) and quiescence (telogen), in which a large number of molecular signals participate. Anagen is characterized by cell

Received December 12, 2015; accepted March 8, 2016

Correspondence: yumei@mail.hzau.edu.cn proliferation in the proximal hair follicle epithelium, which is a unique process of programmed organ regenera$\operatorname{tion}^{[2,3]}$. The length of the hair is determined by the duration of anagen, which is dependent upon the incessant proliferation and differentiation of the matrix cells at the follicle base known as transit-amplifying cells ${ }^{[4]}$. Catagen, a highly controlled process of coordinated cell differentiation and apoptosis, is followed by telogen in which the follicles lie dormant and stay in the upper dermis ${ }^{[5]}$. The continuous postnatal follicle growth cycling has drawn public attention, and subsequently the molecular mechanisms by which the follicles regenerate and regress have been elucidated. Previous studies have shown that many signal pathways are involved in the process of the hair growth cycling, including Wnt/ $\beta$-catenin, BMP, FGF and Shh signaling ${ }^{[6-12]}$. Wnt signaling is involved in many aspects of embryonic development as well as maintaining homeostatic self-regeneration in numerous adult tissues ${ }^{[13]}$. $\beta$-catenin, a key signaling molecule in the Wnt/ $\beta$-catenin pathway, is stabilized in the cytoplasm and then enters cell nuclei to mediate the transcription of the target genes by associating with LEF/TCF transcriptional factor when Wnt signaling is activated. In addition, transcriptional activation is influenced by the interaction of $\beta$ catenin with the histone acetyltransferase CBP, the chromatin remodeling SWI/SNF complex and Bc19 bound to pygopus (Pyg) ${ }^{[14]}$. The $\mathrm{Wnt} / \beta$-catenin pathway is vital for hair follicle development during gestation and growth cycling during postnatal life. The Wnt/ $\beta$-catenin signaling is indispensable for the initiation and maintenance of the hair follicle placodes by cooperation with EDA/EDAR/NF- $\kappa B$ signaling ${ }^{[15-17]}$. Transient activation of $\beta$-catenin in telogen follicles triggers the subsequent anagen, indicating $\mathrm{Wnt} / \beta$-catenin signaling is crucial for the hair follicle postnatal growth cycling ${ }^{[18]}$. Recent research has revealed how Wnt/ $\beta$-catenin signaling can maintain hair-inducing activity of the dermal papilla, the dermal organizing center, in anagen using a skin 
reconstitution assay ${ }^{[19,20]}$. Importantly, Wnt $/ \beta$-catenin signaling is involved in both hair follicle formation and hair growth in the adult.

MicroRNAs (miRNAs), small non-coding RNAs, are single strand molecules of about $22 \mathrm{nt}$ in length processed by Dicer from hairpin precursors and exert their function by targeting mRNA for cleavage or inhibiting translation ${ }^{[21-24]}$. Most of miRNAs are highly conserved and have been implicated in participating in every aspect of biological processes, including embryonic development, apoptosis and proliferation, myogenesis and cardiogenesis ${ }^{[25,26]}$. The analysis of the microRNA expression profiling in skin and hair or feather follicles has been used to discover many important miRNAs related to hair follicle development and growth, especially in sheep, goat and duck ${ }^{[27-30]}$. It has been reported that the miRNA, miR24 promotes hair follicle development by directly targeting Tcf-3 and miR-31 regulates anagen progression and hair follicle formation via inhibiting a number of growth regulatory molecules and cytoskeletal proteins ${ }^{[31,32]}$. Although the role of miRNAs in hair follicle development and growth has recently been explored, the specific molecular mechanisms involved are still unknown. In our previous study, we found that miR-148b was highly expressed in anagen compared with telogen ${ }^{[33]}$. MiR-148b belongs to the miR148/152 family and is involved in the regulation of development of disease and normal tissues ${ }^{[34]}$. It has been reported that miR-148b is required for osteogenesis $^{[35]}$. During adipogenesis the expression of miR-148b is upregulated, which supports a potential role of miR-148b in this process ${ }^{[36]}$. CaMKII $\alpha$ can be downregulated by miR-148b, which suppresses the innate response and Ag-presenting capacity of $\mathrm{DCs}^{[37]}$. Given the high expression of miR-148b in plasma from breast cancer cases, it can be used as a potential biomarker for breast cancer detection ${ }^{[38]}$. However, the specific function and underlying molecular mechanism of miR-148b in hair follicle growth remain unknown.

In this study, we aimed to investigate the role of miR$148 \mathrm{~b}$ in the hair follicle growth cycle. First, the expression pattern of miR-148b in anagen and telogen was detected by qRT-PCR. Also, we explored the effect of miR-148b on the proliferation of hair follicle cells and subsequently investigated the signal pathway by which miR-148b functions. Finally, the targets of miR-148b were predicted and confirmed by a Dual Luciferase system and Western blotting.

\section{Materials and methods}

\subsection{Animal materials and sample collection}

The wool bulbs in different phases (anagen, catagen, telogen) were plucked from three 2-year-old healthy Tibetan sheep supplied by the Agricultural and Animal
Husbandry College of Tibet and immediately homogenized in TRIzol regent (Invitrogen, Carlsbad, CA, USA). After centrifuging at $12000 \mathrm{r} \cdot \mathrm{min}^{-1}$ at $4^{\circ} \mathrm{C}$ for $10 \mathrm{~min}$, the supernatant was preserved at $-80^{\circ} \mathrm{C}$.

\subsection{Cells and cell culture}

Human hair dermal papilla cells (HHDPC) and human hair germinal matrix cells (HHGMC) (ScienCell, San Diego, CA, USA) were cultured with mesenchymal stem cell medium, and maintained in a humidified incubator at $37^{\circ} \mathrm{C}$ supplemented by $5 \% \mathrm{CO}_{2}$. HEK293 and MCF7 cells were cultured in Dulbecco's modified Eagle's medium supplemented with $10 \%$ fetal calf serum (FCS) at $37^{\circ} \mathrm{C}$ in $5 \%$ $\mathrm{CO}_{2}$.

\subsection{Real-time quantitative PCR}

Total mRNA and miRNA were extracted from cells and tissues with TRIzol reagent (Takara Bio In., Dalian, China) and treated with DNase I (Takara Bio In., Dalian, China), the concentration and quality of RNA was assessed through the NanoDrop 2000 and denaturing gel electrophoresis. Reverse transcription was conducted with reverse transcription kit (Takara Bio In., Dalian, China). Random primers, oligo (dT) 12-18 or miRNA specific primers were applied to initiate cDNA synthesis. The real-time quantitative PCR reaction was performed in a LightCycler 480 II (Roche, Basel, Switzerland) system using LightCycler 480 SYBR Green I Master (Roche). The sequences of the miRNA-specific and detection primers are listed in Table 1.

Table 1 The sequences of primers used in the study

\begin{tabular}{ll}
\hline Primer name & \multicolumn{1}{c}{ Sequence 5'-3' $^{\prime}$} \\
\hline miR148b-Forward & GCGTCAGTGCATCACAGAACTTTG \\
GAPDH-Forward & CCTCAAGATTGTCAGCAATGCCT \\
GAPDH-Reverse & TGGTCATAAGTCCCTCCACGAT \\
cycD-Forward & TGTGCATCTACACCGACAACT \\
cycD-Reverse & TTGGGGCTCCTCAGGTTCAG \\
c-jun-Forward & CAGCCAGGTCGGCAGTATAG \\
c-jun-Reverse & GGGACTCTGCCACTTGTCTC \\
PPARD-Forward & GGTCTGGAAAGCAGGGTCAG \\
PPARD-Reverse & ATGGACCTCTACAGGGTGGT \\
$\beta$-catenin-Forward & GCTGGGACCTTGCATAACCT \\
$\beta$-catenin-Reverse & TCCACTGGTGAACCAAGCAT \\
\hline
\end{tabular}

\subsection{Dual-Luciferase reporter assays}

The 3'UTR of NFAT5, Wnt10b housing the miR-148b targeting sequence was cloned into the psiCHECK-2 DualLuciferase reporter plasmid (Promega, Fitchburg, WI, USA). NFAT5 3'UTR was amplified with forward primer: CCGCTCGAGCATAGGCAAACTGTTGGGAGC and 
reverse primer: ATAGTTTAGCGGCCGCAAGGGATGGGGAGACTTGGT. The primer for $3^{\prime} \mathrm{UTR}$ of $\mathrm{Wnt} 10 \mathrm{~b}$ was forward: CCGCTCGAGGCCTTTTCAGCCCTTTGCTC and reverse: ATAGTTTAGCGGCC GCCCACCCTTCCTGCTGAAGAA. The accuracy of the plasmid inserted sequences was validated by complete sequencing analysis. HEK293 cells were plated in growth medium without antibiotics before transfection. When seed cells were $70 \%-90 \%$ confluent, they were transfected. MiRNA mimics or NC-nonspecific miRNA were transfected using the Lipofectamine 2000 (Invitrogen, Carlsbad, CA, USA), with Opti-MEM Reduced-Serum Medium diluting Lipofectamine 2000 and vectors. The transfection procedure was carried out according to the manufacturer's instructions with Lipofectamine 2000 Reagent. The miRNA mimics were synthesized as duplexes. The miRNA mimic sequence for hsa-miR148b, AAGUUCUGUUAUACACUCAGGC and a scrambled sequence, UUCUCCGAACGUGUCACGUTT as negative control (NC) were constructed. The miRNA mimics and 3'UTR Dual-Luciferase vector were cotransfected into cells with Lipofectamine 2000. TOPFlash and FOPFlash (Millipore, Billerica, MA, USA) were transfected into HEK293 with miRNA mimics, respectively. The growth medium was refreshed $6 \mathrm{~h}$ after transfection. Luciferase activity was measured using the Dual-Luciferase reporter assay system (Promega, Fichburg, WI, USA) when cells were cultured for $24 \mathrm{~h}$.

\subsection{Cell proliferation and analysis}

\subsection{1 xCELLigence System real-time analysis}

Approximately 3000 HHDPCs were seeded on a 16-well E-Plate and then grown for $24 \mathrm{~h}$. When the cell index reached 1.0-1.5, miR-148b mimics were transfected with Lipofectamine 2000. Fresh growth medium was used to culture cells after transfection for 5-6 h. Cell growth and proliferation were monitored by xCELLigence RTCA DP instrument (Roche).

\subsubsection{EdU assay}

HHDPC and HHGMC were cultured with fresh growth medium containing 5-ethynyl-2'-deoxyuridine (EdU) (final concentration, $50 \mu \mathrm{mol} \cdot \mathrm{L}^{-1}$ ) for $2 \mathrm{~h}$ after transfection with miR-148b mimics for $6 \mathrm{~h}$. Cells were fixed with $4 \%$ paraformaldehyde and were stained by either Apollo or Hoechst33342, respectively. The number of DNA stained cells and EdU-positive cells was calculated in randomlyselected pictures, respectively.

\subsection{Western blotting}

Protein lysates were generated from MCF7 cells overexpressing miR-148b with RIPA lysis buffer. After sodium dodecyl sulfate-polyacrylamide gel electrophoresis was performed, protein was transferred to polyvinylidene fluoride membrane (Millipore, Billerica, MA, USA) using Mini Trans-Blot Cell (Bio-Rad, Berkeley, CA, USA). Primary antibody specific for NFAT5 (Sangon Biotech, Shanghai, China; 1:500 dilution) and HRPlabeled anti-rabbit $\operatorname{IgG}$ antibody (BOSTER, Wuhan, China; 1:1000 dilution) were used to detect protein expression.

\subsection{Statistical analysis}

All results are presented as mean \pm S.E.M based on at least three replicates for each treatment. Unpaired Student's $t$ test was used for $P$-value calculations.

\subsection{Ethics standards}

The experiments were performed in accordance with the 1996 Guide for the Care and Use of Laboratory Animals $^{[39]}$. The protocols were approved by the Hubei Province for Biological Studies Animal Care and Use Committee and the Ethics Committee of Huazhong Agricultural University.

\section{Results}

3.1 MiR-148b is significantly upregulated during the wool bulb anagen of Tibetan sheep

Previously, we found through Solexa sequencing that the expression of miR-148b in the anagen of wool bulbs was over 5 times the level in telogen, which indicates a potential role of miR-148b in the wool cycle in Tibetan sheep ${ }^{[28]}$. To confirm the expression profile of miR-148b, we collected the hair bulbs of Tibetan sheep in both anagen and telogen to analyze the expression of the miR-148b in hair bulbs by qRT-PCR. The data showed that the expression of miR-148b was upregulated in the anagen and downregulated in the telogen of Tibetan sheep wool bulb (Fig. 1).

\subsection{Overexpression of miR-148b promotes the proliferation} of the hair follicle cells in vitro

We evaluated the role of miR-148b in the proliferation of human dermal papilla cell and human germinal matrix cells, using the xCELLigence system to measure the proliferation of the dermal papilla cells. The data showed that the growth rate of the dermal papilla cells transfected with miR-148b was higher than when transfected with the $\mathrm{NC}$ sequence (Fig. 2a). Subsequently EdU staining was performed to confirm the proliferation tendency of the human dermal papilla cells and human germinal matrix cells. Similarly, the amount of cells that were in a 


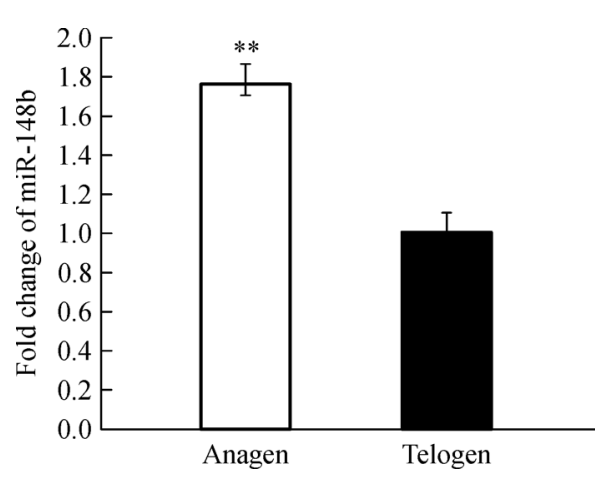

Fig. 1 miR-148b is highly expressed in anagen of the Tibetan sheep hair follicle. The expression of miR-148b of Tibetan sheep wool was quantified by qRT-PCR. Fold change was in relation to the expression in telogen. The results are shown as mean \pm S.E.M. **, $P<0.01$

proliferative state (EdU- positive cells) was significantly increased in miR-148b transfected cells compared with $\mathrm{NC}$ transfected cells (Fig. 2b). These data suggest that miR-148b can promote the proliferation of hair follicle cells.

(a)
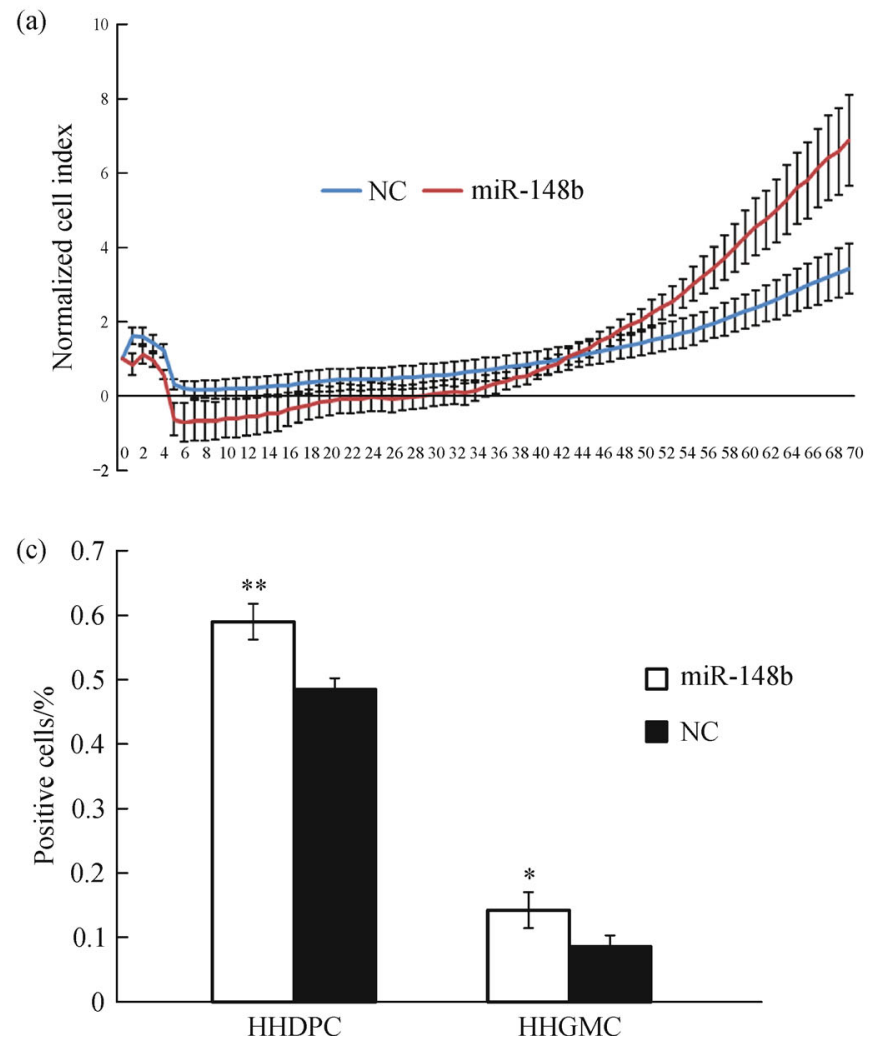

3.3 miR-148b induces the activation of the Wnt signal pathway

To investigate the potential mechanism required for the effect of miR-148b on hair follicle cell proliferation, a Wnt signal reporter system was exploited to detect the activity of the Wnt signal pathway in HEK293 cells via luciferase reporter assay. The luciferase activity in HEK293 cells cotransfected with miR-148b, TOPFlash and PRL-TK was significantly higher than in cells cotransfected with the NC sequence, TOPFlash vector and PRL-TK. However, there was no significant difference between them using the TOPFlash instead of FOPFlash (Fig. 3a). Thus, the result indicates that overexpression of miR-148b activates the Wnt signal pathway.

Subsequently, qRT-PCR was conducted to analyze the effect of miR-148b on cell cycle relevant factors in the Wnt signal pathway in human dermal papilla. $\beta$-catenin, a vital factor in the Wnt pathway, was upregulated in miR-148btreated HHDPC (Fig. 3c). Furthermore, compared to the $\mathrm{NC}$ transfected HHDPC, the downstream genes of the Wnt pathway, such as $c y c D, c-j u n$ and Peroxisome ProliferatorActivated Receptor Delta (PPARD) that are directly

(b) HHDPC
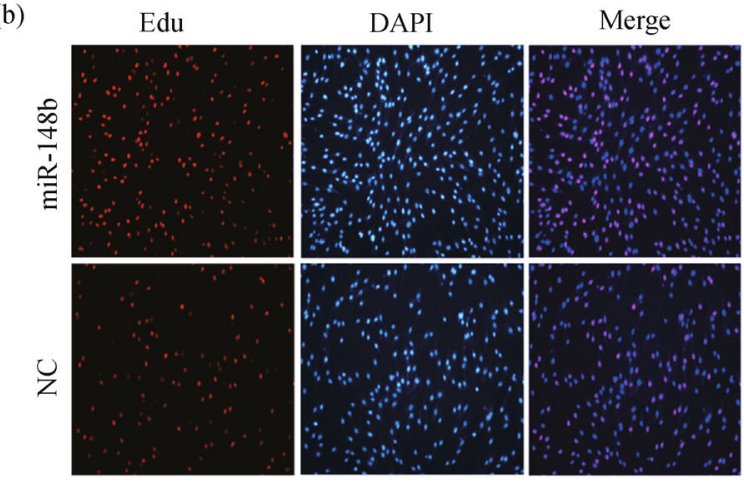

HHGMC
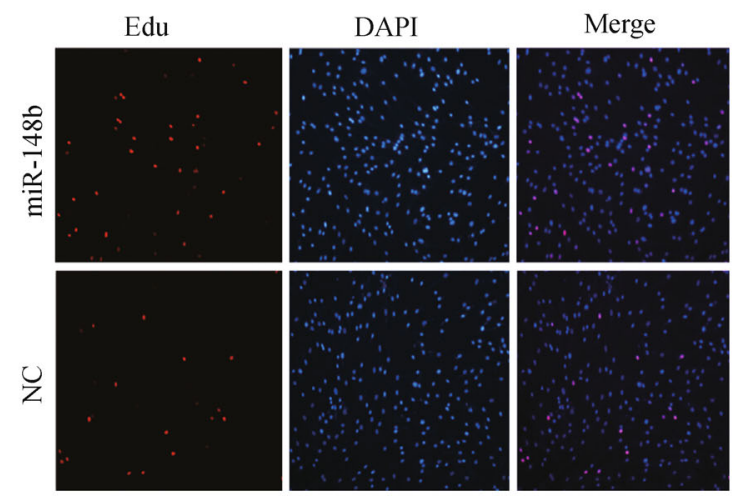

Fig. 2 miR-148b promotes proliferation of the human hair follicle cells. Human hair dermal papillae cells (HHDPC) and human hair germinal matrix cells (HHGMC) were transfected with miR-148b mimics or scrambled NC. (a) HHDPC growth dynamics were constantly monitored with the xCELLigence system; (b) HHDPC and HHGMC transfected with miR-148b were stained with Edu. Cells positive for both Edu and DAPI were merged in pink (Merge); (c) the percentage of dual positive cells was determined. The results are shown as mean \pm S.E.M. $*, P<0.05 ; * *, P<0.01$. 
(a)

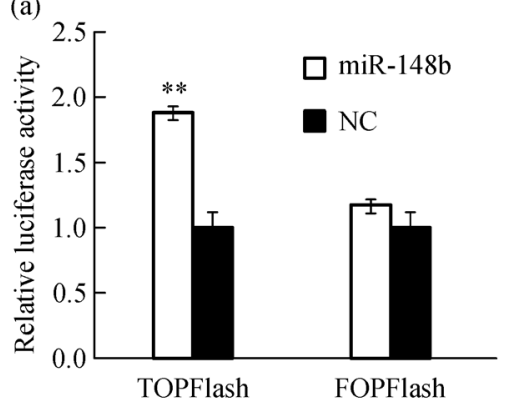

(c)

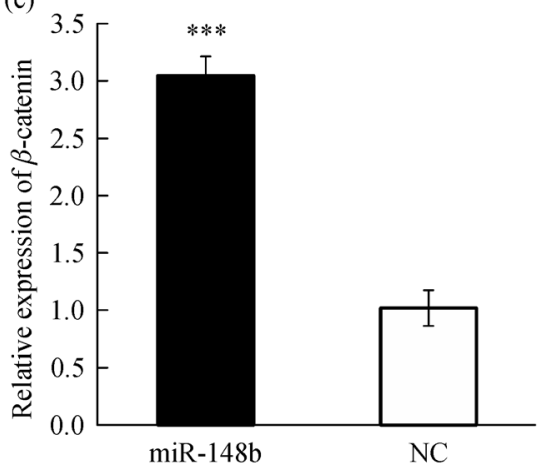

(b)

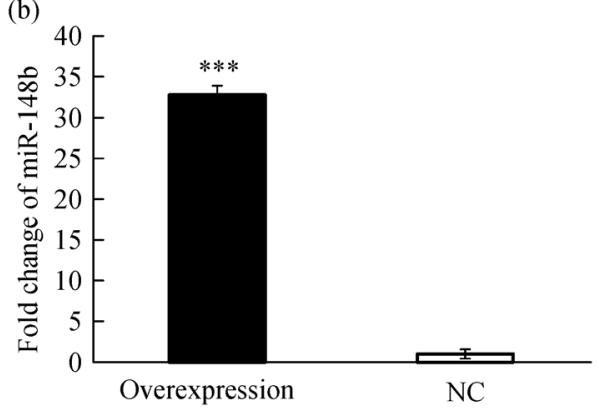

(d)

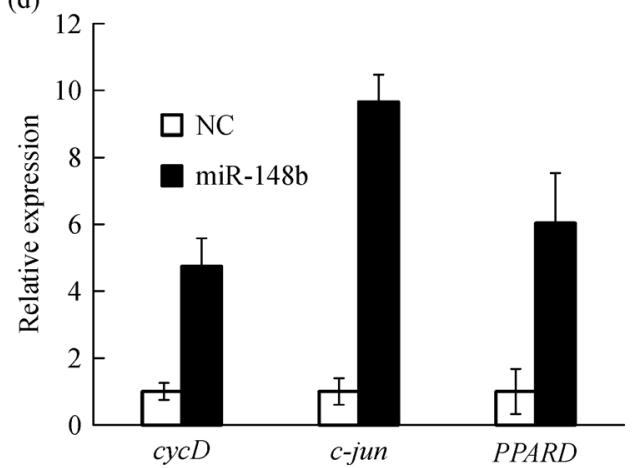

Fig. 3 miR-148b activated Wnt signal pathway. (a) miR-148b mimics or NC were cotransfected with TOPFlash and PRL-TK plasmid or FOPFlash and PRL-TK plasmid, respectively, into HEK293 cells. Normalized luciferase activity was collected; (b) after transfection with miR-148b to HHDPC, cells were analyzed by qRT-PCR for detection of miR-148b. The expression of miR-148b was normalized to U6; (c) the expression of $\beta$-catenin in HHDPC overexpressing miR-148b was determined through qRT-PCR, and its expression was corrected to GAPDH; (d) the factors downstream of Wnt signal pathway, $c y c D, c-j u n$ and PPARD, were detected by qRT-PCR. Their expression levels were normalized to GAPDH. Results are presented as mean \pm S.E.M (three independent replicates per group). ${ }^{*}, P<0.05$; **, $P<0.01 ; * * *, P<0.0001$.

related to cell proliferation, were positively regulated significantly in HHDPC with miR-148b overexpression (Fig. 3d). Thus, collectively,overexpression of miR-148b upregulates the expression of genes, including $c y c D, c-j u n$ and PPARD, through the activation of the Wnt signal pathway.

\subsection{NFAT5 and Wnt10b were direct targets of miR-148b}

To understand the mechanism by which miR-148b functions, we predicted the potential target genes of miR$148 \mathrm{~b}$ by the bioinformatic tool, Targetscan. Of the target genes, NFAT5 and Wnt $10 \mathrm{~b}$ participated in Wnt signaling and were related to cell proliferation. The effect of miR$148 \mathrm{~b}$ on NFAT5 and Wnt10b was validated by a DualLuciferase reporter system in HEK293 cells transfected with constructs containing the corresponding $3^{\prime} \mathrm{UTR}$. We found that in comparison to NC-treated HEK293 the luciferase activity was significantly inhibited in HEK293 cells transfected with miR-148b mimics, indicating that miR-148b reduced the expression of NFAT5 and Wnt $10 \mathrm{~b}$ by binding to their 3'UTRs (Fig. 4a). In addition, Western blotting was performed with the protein extracted from MCF7 overexpressing miR-148b/NC to confirm the results found above. Consistently, there was a significant decrease of NFAT5 protein in MCF7 cells with miR-148b mimics
(Fig. 4b, Fig. 4c). Together, these results revealed that miR-148b can target NFAT5 and Wnt $10 b$ to indirectly mediate the activity of the Wnt signal pathway or cell proliferation.

\section{Discussion}

Hair follicles are continually regenerating miniorgans undergoing the processes of proliferation, differentiation and apoptosis throughout an animal's lifetime. Anagen of the hair follicle is characterized by the activation of hair follicle stem cell clustering in the hair bulge area and subsequently the proliferation of the matrix cells, known as transit-amplifying cells, before differentiation ${ }^{[40]}$. MiRNAs control expression of protein coding genes by translational inhibition and mRNA destabilization to regulate the biological process ${ }^{[25]}$. Dicer required for processing of miRNA and assembling into the RNAinduced silencing complex is essential for maintenance of the normal hair follicle, which indicates the necessity for miRNA in development and growth of hair follicles ${ }^{[41]}$. MiR-148b was found to participate in osteogenesis, adipogenesis and be dysregulated in many cancers ${ }^{[35,36]}$. It was also shown that miR-148b is significantly upregulated in wool follicle anagen of Tibetan sheep. 


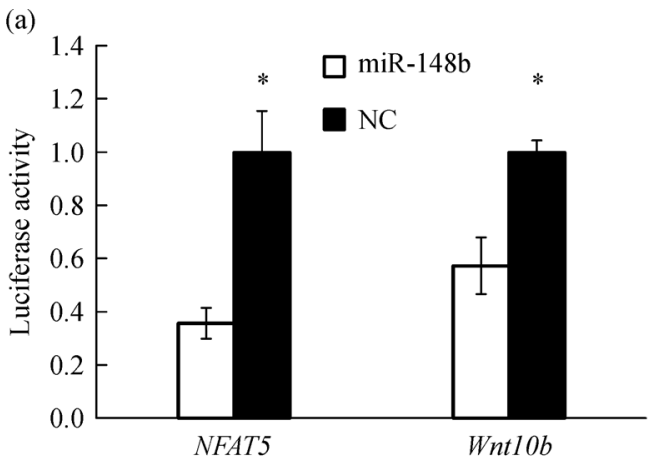

(b)

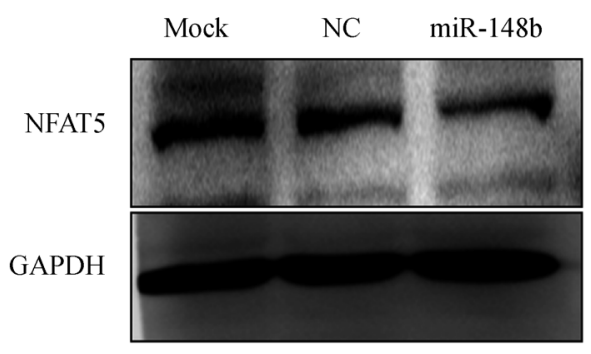

(c)

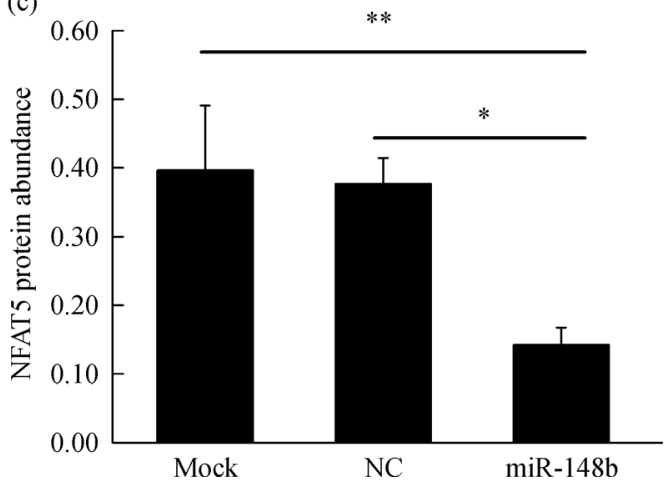

Fig. 4 NFAT5 and Wnt10b were identified as the targets of miR-148b. (a) The 3'URT of NFAT5 and Wnt10b were inserted into the dualluciferase reporter vector psi-CHECK2 at the end of the Renllia luciferase gene $(h R l u c)$. miR-148b mimics or NC were cotransfected with psi-CHECK2 containing 3'UTR mentioned above, into HEK293 cells. The constitutive firefly luciferase (hluc ${ }^{+}$) expression was used as an internal normalized control, and normalized Renllia luciferase activity was assayed; (b) NFAT5 protein expression in MCF7 cells was determined after transfection with miR-148b into the MCF7 cells for $36 \mathrm{~h}$. Comparable GAPDH levels served as a loading control during western blotting; (c) results of NFAT5 protein expression in MCF7 cells are presented as mean \pm S.E.M (three independent replicates per group). *, $P<0.05 ; * *, P<0.01$.

Subsequently, we demonstrated that miR-148b promotes the proliferation of HHDPC and HHGMC. In contrast, Zhang et al. reported that miR-148b suppressed hepatocelluar carcinoma growth by targeting the Wnt/ $\beta$-catenin pathway $^{[42]}$. Likewise, there are differing results for the expression of miR-148b in specific cancers, such as downregulation in colorectal cancer, gastric cancer and breast cancer, conversely upregulation in ovarian carcinoma ${ }^{[43-46]}$. The conflicting results may be due to the difference in functions of miR-148b in various types of tissues. Therefore, miR-148b probably regulates proliferation of the hair follicle cells, especially hair matrix cells, to increase the growth rate of the hair follicle.

The Wnt pathway, initiated after the combination of Wnt family members and respective receptors/coreceptors, is needed for both development and homeostasis in tissues by bridging short range cell-cell communication ${ }^{[13,47]}$. The canonical $\mathrm{Wnt} / \beta$-catenin pathway has been identified to contribute to development and growth of the hair follicle. $\beta$-catenin, a critical factor in the canonical Wnt pathway, is necessary for the induction and morphogenesis of the hair follicle by determining the fate of embryonic epidermis $^{[48,49]}$. $\beta$-catenin in epidermis and hair follicle dermal papilla is required to promote the proliferation of progenitors to induce or maintain the anagen of the hair follicle ${ }^{[50-52]}$. When the $\mathrm{Wnt} / \beta$-catenin pathway was activated, the luciferase activity of TOPFlash containing $\mathrm{T}$ cell binding site ( $\mathrm{Tcf}$ ) would be significantly increased compared with that in FOPFlash with mutated Tcf binding site. As expected, our results from the TOPFlash luciferase reporter system indicated that miR-148b could enhance the activity of the $\mathrm{Wnt} / \beta$-catenin pathway, revealing a potential role for miR-148b in hair follicle growth cycling. Then, factors functioning in $\mathrm{Wnt} / \beta$-catenin pathway, including $\beta$-catenin, cycD, c-jun and PPARD, were found to be upregulated in HHDPC overexpressing miR-148b, further substantiating that miR-148b can positively regulate the $\mathrm{Wnt} / \beta$-catenin pathway. Previous reports indicate that $\beta$ catenin signaling in both epidermis and dermal papilla is essential for maintenance of proliferation in anagen hair follicles ${ }^{[49,51]}$. Therefore miR-148b was considered to stimulate the progress of the hair follicle anagen by means of activating the Wnt/ $\beta$-catenin pathway to promote proliferation in vivo.

To reveal the underlying mechanism used by miR-148b, putative target genes (Wnt10b and NFAT5) of miR-148b 
were indentified. The luciferase reporter results revealed miR-148b could bind to the $3^{\prime}$ UTRs of the two genes. In addition, the expression of the NFAT5 protein was significantly repressed by overexpression of miR-148b. Our results indicate that miR-148b can bind to Wnt $10 \mathrm{~b}$ and NFAT5 mRNAs to suppress their translation directly.

The Wnt/ $\beta$-catenin pathway engages in wide range of biological events, including cell proliferation, terminal differentiation and maintenance or activation of stem cells $^{[13]}$. However, the precise function of $\mathrm{Wnt} / \beta$-catenin has yet to be fully known. Wnt $10 b$ promotes differentiation in hair follicles to influence hair shaft growth ${ }^{[53,54]}$. Also, it was reported that $W n t 10 b$ suppressed proliferation and promoted the differentiation in epithelial stem/progenitor cells and melanocytes in the skin through activating canonical Wnt signaling ${ }^{[5,56]}$. MiR-148b may promote the growth rate of hair follicle by downregualtion of $W n t 10 b$ in hair follicle cells that rapidly proliferates in anagen. $\beta$ catenin contains several functional domains, including the $\mathrm{N}$-terminal domain responsible for $\beta$-catenin stability, the central armadillo domains that bind to TCF/LEF and Ecadherin and $\mathrm{C}$-terminal domain that interacts with transcriptional mediator complex ${ }^{[54,57]}$. Considering various aspects of $\mathrm{Wnt} / \beta$-catenin signaling, not only are $\beta$ catenin nuclear localization and its interaction with TCF important, but also the combination of $\beta$-catenin and transcriptional mediators plays a crucial role ${ }^{[58,59]}$. NFAT5 can interact with the $\mathrm{C}$-terminal transactivation domain of $\beta$-catenin to block its interaction with CBP to inhibit CBPmediated $\beta$-catenin acetylation and ultimately inhibit the canonical Wnt pathway ${ }^{[58]}$. The interaction between NFAT5 and $\beta$-catenin can be counteracted by miR-148b and then leads to acetylation of $\beta$-catenin. Subsequently, the acetylated $\beta$-catenin activates the $\mathrm{Wnt} / \beta$-catenin pathway to increase the expression of downstream genes, $c y c D$, c-jun and PPARD which are related to the cell cycle. Although, the role of NFAT5 in hair follicles still remains unclear, we have provided evidence that miR-148b targets Wnt $10 b$ and NFAT5 to regulate the proliferation of hair follicle cells.

\section{Conclusions}

This study found that miR-148b was highly expressed in the anagen of hair follicle and could promote proliferation of hair follicle cells. In addition, we provided data that indicates miR-148b could activate $\mathrm{Wnt} / \beta$-catenin signaling by upregulating the expression of $\beta$-catenin, $c y c D, c$-jun and PPARD, which may be mediated by targeting NFAT5 and $W n t 10 b$. These results suggested that miR-148b might be important for increased proliferation of hair follicle in anagen.

Acknowledgements This work was supported by grants from the National High Technology Research and Development Program of China
(2013AA102506), and Regional Project of National Natural Science Foundation of China (31360534). We would like to thank Dr. Xiaohui Tang for the help in sample collection.

Compliance with ethics guidelines Wanbao Yang, Qinqun $\mathrm{Li}$, Bo Su, and Mei Yu declare that they have no conflict of interest or financial conflicts to disclose.

All applicable institutional and national guidelines for the care and use of animals were followed.

\section{References}

1. Schneider M R, Schmidt-Ullrich R, Paus R. The hair follicle as a dynamic miniorgan. Current Biology, 2009, 19(3): R132-R142

2. Botchkarev V A, Paus R. Molecular biology of hair morphogenesis: development and cycling. Journal of Experimental Zoology Part B: Molecular and Developmental Evolution, 2003, 298B(1): 164-180

3. Oshima H, Rochat A, Kedzia C, Kobayashi K, Barrandon Y. Morphogenesis and renewal of hair follicles from adult multipotent stem cells. Cell, 2001, 104(2): 233-245

4. Alonso L, Elaine F. The hair cycle. Journal of Cell Science, 2006, 119(3): 391-393

5. Stenn K S, Paus R. Controls of hair follicle cycling. Physiological Reviews, 2001, 1:449-494

6. Yuhki M, Yamada M, Kawano M, Iwasato T, Itohara S, Yoshida H, Ogawa M, Mishina Y. BMPR1A signaling is necessary for hair follicle cycling and hair shaft differentiation in mice. Development, 2004, 131(8): 1825-1833

7. Plikus M V, Mayer J A, de la Cruz D, Baker R E, Maini P K, Maxson R, Chuong C M. Cyclic dermal BMP signalling regulates stem cell activation during hair regeneration. Nature, 2008, 451 (7176): 340-344

8. Botchkarev V A, Sharov A A. BMP signaling in the control of skin development and hair follicle growth. Differentiation, 2004, 72(910): 512-526

9. Kawano M, Komi-Kuramochi A, Asada M, Suzuki M, Oki J, Jiang J, Imamura T. Comprehensive analysis of FGF and FGFR expression in skin: FGF18 is highly expressed in hair follicles andcapable of inducing anagen from telogen stage hair follicles. Journal of Investigative Dermatology, 2005, 124(5): 877-885

10. Suzuki S, Ota Y, Ozawa K, Imamura T. Dual-mode regulation of hair growth cycle by two Fgf-5 gene products. Journal of Investigative Dermatology, 2000, 114(3): 456-463

11. Sato N, Leopold P L, Crystal R G. Induction of the hair growth phase in postnatal mice by localized transient expression of Sonic hedgehog. Journal of Clinical Investigation, 1999, 104(7): 855-864

12. Van Mater D, Kolligs F T, Dlugosz A A, Fearon E R. Transient activation of $\beta$-catenin signaling in cutaneous keratinocytes is sufficient to trigger the active growth phase of the hair cycle in mice. Genes \& Development, 2003, 17(10): 1219-1224

13. Gough N R. Focus issue: Wnt and $\beta$-catenin signaling in development and disease. Science Signaling, 2012, 5(206): eg2

14. Huelsken J, Juergen B. The Wntsignaling pathway. Journal of Cell Science, 2002, 115(21): 3977-3978

15. Andl T, Reddy S T, Gaddapara T, Millar S E. WNT signals are required for the initiation of hair follicle development. Develop- 
mental Cell, 2002, 2(5): 643-653

16. Zhang Y, Tomann P, Andl T, Gallant N M, Huelsken J, Jerchow B, Birchmeier W, Paus R, Piccolo S, Mikkola M L, Morrisey E E, Overbeek P A, Scheidereit C, Millar S E, Schmidt-Ullrich R. Reciprocal requirement forEDA/EDAR/NF-B and Wnt/ $\beta$-catenin signaling pathways in hair follicle induction. Developmental Cell, 2009, 17(1): 49-61

17. Fu J, Hsu W. Epidermal Wnt controls hair follicle induction by orchestrating dynamic signaling crosstalk between the epidermis and dermis. Journal of Investigative Dermatology, 2013, 133(4): 890-898

18. Van Mater D, Kolligs F T, Dlugosz A A, Fearon E R. Transient activation of $\beta$-catenin signaling in cutaneous keratinocytes is sufficient to trigger the active growth phase of the hair cycle in mice. Genes \& Development, 2003, 17(10): 1219-1224

19. Kishimoto J, Burgeson R E, Morgan B A. Wnt signaling maintains the hair-inducing activity of the dermal papilla. Genes \& Development, 2000, 14(10): 1181-1185

20. Soma T, Fujiwara S, Shirakata Y, Hashimoto K, Kishimoto J. Hairinducing ability of human dermal papilla cells cultured under Wnt/ $\beta$-catenin signalling activation. Experimental Dermatology, 2012, 21(4): 307-309

21. Bartel D P. MicroRNAs: genomics, biogenesis, mechanism, and function. Cell, 2004, 116(2): 281-297

22. Kidner C A, Martienssen R A. The developmental role of microRNA in plants. Current Opinion in Plant Biology, 2005, 8 (1): $38-44$

23. Ambros V, Bartel B, Bartel D P, Burge C B, Carrington J C, Chen X, Dreyfuss G, Eddy S R, Griffiths-Jones S, Marshall M, Matzke M, Ruvkun G, Tuschl T. A uniform system for microRNA annotation. RNA, 2003, 9(3): 277-279

24. Hutvagner G, Zamore P D. A microRNA in a multiple- turnover RNAi enzyme complex. Science, 2002, 297(5589): 2056-2060

25. Bushati N, Cohen S M. microRNA Functions. Annual Review of Cell and Developmental Biology, 2007, 23(1): 175-205

26. Kloosterman W P, Plasterk R H. The diverse functions of microRNAs in animal development and disease. Developmental Cell, 2006, 11(4): 441-450

27. Zhang L, Nie Q, Su Y, Xie X, Luo W, Jia X, Zhang X. MicroRNA profile analysis on duck feather follicle and skin with highthroughput sequencing technology. Gene, 2013, 519(1): 77-81

28. Liu G, Liu R, Li Q, Tang X, Yu M, Li X, Cao J, Zhao S. Identification of microRNAs in wool follicles during anagen, catagen, and telogenphases in Tibetan sheep. PLoS ONE, 2013, 8 (10): e 77801

29. Liu Z, Xiao H, Li H, Zhao Y, Lai S, Yu X, Cai T, Du C, Zhang W, Li J. Identification of conserved and novel microRNAs in cashmeregoat skin by deepsequencing. PLoS ONE, 2012, 7(12): e50001

30. Wenguang Z, Jianghong W, Jinquan L, Yashizawa M.A subset of skin-expressed microRNAs with possible roles in goat and sheep hair growth based on expression profiling of mammalian microRNAs.Omics: aJournal of Integrative Biology, 2007, 1(4):385-396

31. Mardaryev A N, Ahmed M I, Vlahov N V, Fessing M Y, Gill J H, Sharov A A, Botchkareva N V. Micro-RNA-31 controls hair cycleassociated changes in gene expression programs of the skin and hair follicle. FASEB Journal, 2010, 24(10): 3869-3881
32. Amelio I, Lena A M, Bonanno E, Melino G, Candi E. miR-24 affects hair follicle morphogenesis targeting Tcf-3. Cell Death \& Disease, 2013, 4(11): e922

33. Liu G, Liu R, Li Q, Tang X, Yu M, Li X, Cao J, Zhao S. Identification of microRNAs in wool follicles during anagen, catagen, and telogen phases in Tibetan sheep. PLoS ONE, 2013, 8 (10): e77801

34. Chen Y, Song Y X, Wang Z N. The microRNA-148/152 family: multi-faceted players. Molecular Cancer, 2013, 12(1): 43

35. Schoolmeesters A, Eklund T, Leake D, Vermeulen A, Smith Q, Force Aldred S, Fedorov Y. Functional profiling reveals critical role for miRNA in differentiation of human mesenchymal stem cells. PLoS ONE, 2009, 4(5): e5605

36. John E, Wienecke-Baldacchino $\mathrm{A}$, Liivrand $\mathrm{M}$, Heinäniemi $\mathrm{M}$, Carlberg C, Sinkkonen L. Dataset integration identifies transcriptional regulation of microRNA genes by PPARy in differentiating mouse 3T3-L1 adipocytes. Nucleic Acids Research, 2012, 40(10): 4446-4460

37. Liu X, Zhan Z, Xu L, Ma F, Li D, Guo Z, Li N, Cao X. MicroRNA$148 \mathrm{~b} / 152$ impair innate response and antigen presentation of TLRtriggered dendritic cells by targeting CaMKII $\alpha$. Journal of Immunology, 2010, 185(12): 7244-7251

38. Shen J, Hu Q, Schrauder M, Yan L, Wang D, Medico L, Guo Y, Yao S, Zhu Q, Liu B, Qin M, Beckmann M W, Fasching P A, Strick R, Johnson C S, Ambrosone C B, Zhao H, Liu S.Circulating miR-148b and miR-133a as biomarkers for breast cancer detection.National Institutes of Health, 2014, 5(14): 5284-5294

39. Clark J D, Gebhart G F, Gonder J C, Keeling M E, Kohn D F. The 1996 guide for the care and use of laboratory animals. ILAR Journal, 1997, 38(1): 41-48

40. Paus R, Foitzik K. In search of "hair cycle clock": a guided tour. Differentiation, 2004, 72(9-10): 489-511

41. Andl T, Murchison E P, Liu F, Zhang Y, Yunta-Gonzalez M, Tobias J W, Andl C D, Seykora J T, Hannon G J, Millar S E. The miRNAprocessing enzyme dicer is essential for the morphogenesis and maintenance of hair follicle. Current Biology, 2006, 16(10): 10411049

42. Zhang J G, Shi Y, Hong D F, Song M, Huang D, Wang C Y, Zhao G. MiR-148b suppresses cell proliferation and invasion in hepatocellular carcinoma by targeting WNT1/ $\beta$-catenin pathway. Scientific Reports, 2015, 5: 8087

43. Song Y, Xu Y, Wang Z, Chen Y, Yue Z, Gao P, Xing C, Xu H. MicroRNA-148b suppresses cell growth by targeting cholecystokinin-2 receptor in colorectal cancer. International Journal of Cancer, 2012, 131(5): 1042-1051

44. Song Y X, Yue Z Y, Wang Z N, Xu Y Y, Luo Y, Xu H M, Zhang X, Jiang L, Xing C Z, Zhang Y. MicroRNA-148b is frequently downregulated in gastric cancer and acts as a tumor suppressor by inhibiting cell proliferation. Molecular Cancer, 2011, 10(1): 1

45. Cimino D, De Pittà C, Orso F, Zampini M, Casara S, Penna E, Quaglino E, Forni M, Damasco C, Pinatel E, Ponzone R, Romualdi C, Brisken C, De Bortoli M, Biglia N, Provero P, Lanfranchi G, Taverna D. miR148b is a major coordinator of breast cancer progression in a relapse-associated microRNA signature by targeting ITGA5, ROCK1, PIK3CA, NRAS, and CSF1. FASEB Journal, 2013, 27(3): 1223-1235 
46. Chang H, Zhou X, Wang Z N, Song Y X, Zhao F, Gao P, Chiang Y, $\mathrm{Xu} \mathrm{H}$ M. Increased expression of miR-148b in ovarian carcinoma and its clinical significance. Molecular Medicine Reports, 2012, 5 (5): 1277-1280

47. Yu J, Virshup D M. Updating the Wnt pathways. Bioscience Reports, 2014, 34(5): e00142

48. Gat U, DasGupta R, Degenstein L, Fuchs E. De novo hair follicle morphogenesis and hair tumors in mice expressing a truncated $\beta$ catenin in skin. Cell, 1998, 95(5): 605-614

49. Zhang Y, Andl T, Yang S H, Teta M, Liu F, Seykora J T, Tobias J W, Piccolo S, Schmidt-Ullrich R, Nagy A, Taketo M M, Dlugosz A A, Millar S E.Activation of -catenin signaling programs embryonic epidermis to hair follicle fate. National Institutes of Health, 2008, 135(12):2161-2172

50. Enshell-Seijffers D, Lindon C, Kashiwagi M, Morgan B A. bcatenin activity in the dermal papilla regulates morphogenesis and regeneration of hair. Developmental Cell, 2010, 18(4): 633-642

51. Huelsken J, Vogel R, Erdmann B, Cotsarelis G, Birchmeier W. bcatenin controls hair follicle morphogenesis and stem cell differentiation in the skin. Cell, 2001, 105(4): 533-545

52. Choi Y S, Zhang Y, Xu M, Yang Y, Ito M, Peng T, Cui Z, Nagy A, Hadjantonakis A K, Lang R A, Cotsarelis G, Andl T, Morrisey E E, Millar S E. Distinct functions for Wnt $/ \beta$-catenin in hair follicle stem cell proliferation and survival and interfollicularepidermal homeostasis. Cell Stem Cell, 2013, 13(6): 720-733
53. Ouji Y, Yoshikawa M, Moriya K, Ishizaka S.Effects of Wnt-10b on hair shaft growth in hair follicle cultures. Biochemical and Biophysical Communications, 2007, 359(3):516-522

54. Ouji Y, Yoshikawa M, Moriya K, Nishiofuku M, Matsuda R, Ishizaka S. Wnt-10b, uniquely among Wnts, promotes epithelial differentiation and shaft growth. Biochemical and Biophysical Research Communications, 2008, 367(2): 299-304

55. Ouji Y, Yoshikawa M, Nishiofuku M, Ouji-Sageshima N, Kubo A, Ishizaka S. Effects of Wnt-10b on proliferation and differentiation of adult murine skin-derived CD34 and CD49f double-positive cells. Journal of Bioscience and Bioengineering, 2010, 110(2): 217-222

56. Ye J X, Yang T, Guo H Y, Tang Y H, Deng F, Li Y H, Xing Y Z, Yang L, Yang K. Wnt10b promotes differentiation of mouse hair follicle melanocytes. International Journal of Medical Sciences, 2013, 10(6): 691-698

57. Hecht A, Vleminckx K, Stemmler M P, Van Roy F, Kemler R. The p300/CBP acetyltransferases function as transcriptional coactivators of $\beta$-catenin in vertebrates. EMBO Journal, 2000, 19(8): 1839-1850

58. Wang Q, Zhou Y, Rychahou P, Liu C, Weiss H L, Evers B M. NFAT5 represses canonical Wnt signaling via inhibition of $\beta$-catenin acetylation and participates in regulating intestinal cell differentiation. Cell Death \& Disease, 2013, 4(6): e671

59. Giles R H, Van Es J H, Clevers H. Caught up in a Wnt storm: Wnt signaling in cancer. Biochimica et Biophysica Acta (BBA)Reviewson Cancer, 2003, 1653(1): 1-24 